\title{
SP1 upregulated FoxO3a promotes tumor progression in colorectal cancer
}

\author{
YIYI YU* ${ }^{*}$ KE PENG ${ }^{*}$, HONG LI ${ }^{*}$, RONGYUAN ZHUANG, YAN WANG, WEI LI, SHAN YU, \\ LI LIANG, XIAOJING XU and TIANSHU LIU
}

Department of Oncology, Zhongshan Hospital, Fudan University, Shanghai 200032, P.R. China

Received October 3, 2017; Accepted March 8, 2018

DOI: $10.3892 / o r .2018 .6323$

\begin{abstract}
FoxO transcription factors are important regulators of cell survival in response to a variety of stress stimuli and play vital functions in tumor progression. However, the functions and underlying regulators of FoxO3a in colorectal cancer (CRC) have not been fully elucidated. The aim of the current study was to identify the functions of FoxO3a in $\mathrm{CRC}$ and characterize the transcription elements within the promoter region of FoxO3a. The expression of FoxO3a was upregulated in response to hypoxic and oxidative stress stimuli. Furthermore, knockdown of FoxO3a significantly reduced cell proliferation and migration ability, while it promoted the response to cetuximab treatment. In addition, it was revealed that knockdown of FoxO3a reduced tumor progression in vivo. A mechanistic study found that plenty of putative SP1 sites were identified in the FoxO3a promoter. Luciferase reporter assay revealed that a region corresponding to the SP1 binding sites located between $-2,000$ and $-1,037$ bp of FoxO3a promoter was essential for the transcriptional activity. Co-transfection of a SP1 expression vector with the reporter constructs markedly increased luciferase activity. Collectively, these results indicated that SP1-dependent promoter elements drive FoxO3a gene transcription in colorectal CRC, and indicated that SP1 upregulated FoxO3a is critical for CRC progression.
\end{abstract}

\section{Introduction}

Since colorectal cancer (CRC) is the third most common cause of cancer-related deaths worldwide it has attracted attention in recent years (1). Research has revealed that stresses including oxidative stress and low nutrient availability are intrinsic of the tumor microenvironment and have significant roles in CRC development, progression and therapy $(2,3)$.

Correspondence to: Professor Tianshu Liu, Zhongshan Hospital, Fudan University, 180 Fenglin Road, Shanghai 200032, P.R. China E-mail: liutianshu1969@126.com

*Contributed equally

Key words: colorectal cancer, FoxO3a, SP1, transcription, stimuli
FoxO3a as a transcription factor is evolutionarily conserved. It is involved in many pivotal biological processes, such as cell cycle regulation, DNA damage repair, vascular development, reactive oxygen species detoxification pathways, longevity and regulation of immune responses (4-9). In cancer, previous studies have revealed that FoxO3a is a tumor suppressor gene and controls diverse genetic pathways $(10,11)$. For example, it has been found that decreased expression of FoxO3a predicted advanced recurrence and poor survival in CRC (12), indicating a tumor-suppressor role for this transcription factor. In addition, recent data indicated that FoxO3a may also function as a metastasis inductor and promote tumor progression in CRC through interaction with $\beta$-catenin (13). These studies indicated that the function of FoxO3a in CRC is highly context dependent, relying on the upstream regulation. However, little research has been conducted concerning the regulation of FoxO3a gene transcription.

SP1 is a zinc finger transcription factor that binds to GC-rich motifs of many promoters. SP1 is involved in many cellular processes, including cell growth, apoptosis, immune response, chromatin remodeling and DNA damage response. It is reported that inhibition of SP1 suppresses colon cancer stem cell growth and induces apoptosis in vitro and in vivo (14). SP1 also has anti-proliferative effects and promotes apoptosis in oral squamous cell carcinoma $(15,16)$. The activity of SP1 is modulated by growth factors, cytokines and tumor promoters (17).

Therefore, in the present study, we performed a functional analysis of FoxO3a promoter and identified the core sequences within the 5 ' regulatory region that critically affected FoxO3a transcriptional activity. Furthermore, plenty of SP1 transcription factor binding sites were found in the core promoter regulatory region and we verified that SP1 played an important function in FoxO3a transcription. Collectively, our findings identified SP1 as a critical transcription factor in regulating FoxO3a expression, which may offer new insights in CRC progression and treatment.

\section{Materials and methods}

Cell culture and treatment. Caco2 and HT29 cell lines were obtained from the Cell Bank of the Chinese Academy of Sciences (Shanghai, China). Caco2 cells were maintained in Dulbecco's modified Eagle's medium (DMEM; HyClone 
Table I. Sequences of primers used for PCR in the present study.

Primer name

Primer sequences

FoxO3a primer-F

FoxO3a primer-R

FoxO3a promoter-WT-F

FoxO3a promoter-WT-R

FoxO3a promoter-1037-F

FoxO3a promoter-513-F

FoxO3a promoter-62-F

FoxO3a promoter-62-R

Human-SP1-F

Human-SP1-R

Human-TFAP2A-F

Human-TFAP2A-R
GGTGCTGTATAGGTGCTTTCT

AAAGGTGGTCCCAACTATTCC

CCGGAATTCATAAGGACTTGTGCAGATGTTTTT

CGACGCGTCAGGAGGACCTGAAGACGTG

CCGCTCGAGTGAACTAGTGTGTAGACTTTTGGTGTG

CCGCTCGAGACACCGGGGCTGGCCCAGA

TCGAGCGCCCGCCGTCAGCCTAGGTTGAGGCGCCCTGCGTGTGTCTATAACTTTGTG CTGCTGCCGCA

AGCTTGCGGCAGCAGCACAAAGTTATAGACACACGCAGGGCGCCTCAACCTAGGCTG ACGGCGGGCGC

GAGCGACCAAGATCACTCCATG

CCGCTCGAGTCAGAAGCCATTGCCACTGAT

ACTTTGGAAATTGACGGATAATATCA

CCGCTCGAGTCACTTTCTGTGCTTCTCCTCTTT

F, forward; $\mathrm{R}$, reverse.

Laboratories; GE Healthcare, Logan, UT, USA) containing $10 \%$ fetal bovine serum (FBS). The HT29 cells were cultured in RMPI-1640 medium (HyClone Laboratories; GE Healthcare) supplemented with $10 \%$ FBS. Both cell lines were cultured in a $5 \% \mathrm{CO}_{2}$ humidified atmosphere at $37^{\circ} \mathrm{C}$. The cells were treated with $\mathrm{CoCl}_{2}(200 \mu \mathrm{M} / \mathrm{l})$ or $\mathrm{H}_{2} \mathrm{O}_{2}(200 \mu \mathrm{mol} / \mathrm{l})$ for $24 \mathrm{~h}$.

cDNA synthesis and quantitative real-time polymerase chain reaction ( $q R T-P C R)$. Total RNA was extracted from Caco2 and HT29 cell lines using TRIzol reagent (Life Technologies; Thermo Fisher Scientific, Inc., Waltham, MA, USA). Subsequently, mRNA was reverse transcribed using SuperScript First-Strand Synthesis System (Invitrogen; Thermo Fisher Scientific, Inc., Waltham, MA, USA) according to the manufacturer's instructions and then qRT-PCR was performed using SYBR Green reagents (Takara Bio, Inc., Otsu, Japan) containing $100 \mu \mathrm{M}$ final concentration of FoxO3a primers (primer sequences are listed in Table I). PCR conditions were as follows: $95^{\circ} \mathrm{C}$ for $15 \mathrm{sec}$, followed by 40 cycles of $95^{\circ} \mathrm{C}$ for $5 \mathrm{sec}, 60^{\circ} \mathrm{C}$ for $30 \mathrm{sec}$. PCRs were performed in triplicates using the Roche LightCycler 480 II RT-PCR system (Roche diagnostics Nederland BV, Almere, The Netherlands). The expression levels of the target genes were normalized to $\beta$-actin. The relative levels were calculated by the comparative $\mathrm{Ct}(\Delta \Delta \mathrm{Ct})$ method and the relative expression fold $\left(2^{-\Delta \Delta \mathrm{Ct}}\right)$ was calculated (18).

Western blot analysis. Cell samples were collected and lysed in RIPA buffer with protease inhibitor cocktails (Sigma-Aldrich; Merck KGaA, Darmstadt, Germany). Total cell protein extracts $(20 \mu \mathrm{g} /$ lane) were subjected to SDS-PAGE analysis. The membrane was blocked with $5 \%$ milk in TBST before being incubated with FoxO3a antibody (cat. no. 12829; Cell Signaling Technology, Inc., Danvers, MA, USA), SP1 antibody (cat. no. 07-645; EMD Millipore, Billerica, MA, USA) or AP2 antibody (cat. no. H00000160-K; Abnova, Taipei, Taiwan) overnight at $4^{\circ} \mathrm{C}$. The membranes were washed with TBST and incubated with the secondary antibodies (cat. no. sc-2357; Santa Cruz Biotechnology, Inc., Dallas, TX, USA). The immunoreactive proteins were visualized by chemiluminescence reagents (ECL; Amersham Biosciences; GE Healthcare, Chicago, IL, USA).

Lentiviral-vector mediated FoxO3a-knockdown stable cells. The target sequences for FoxO3a were 5'-GCTCTTGGTGGA TCATCAA-3' (FoxO3a-KD1) and 5'-GCATGTTCAATG GGAGCTTGGA-3' (FoxO3a-KD2). Lentiviral vectors with green fluorescent protein (GFP) sequence containing human FoxO3a-shRNA were constructed. All of the constructs were validated by DNA sequencing. The recombinant FoxO3a knockdown and negative control (NC) lentivirus were prepared. Caco2 or HT29 cells were seeded in six-well dishes in antibiotic-free medium 1 day before infection. The cells reached $80-90 \%$ confluency at the time of transfection. The expression of GFP was confirmed using a fluorescence microscope after the lentivirus infection. The culture medium was added with $4 \mu \mathrm{g} / \mathrm{ml}$ puromycin for at least 14 days. The puromycin-resistant cell clones were then amplified in medium containing $2 \mu \mathrm{g} / \mathrm{ml}$ puromycin for 7-9 days. The clones were designated as FoxO3a-KD or NC cells.

Cell proliferation assay, cell migration and cell apoptosis assay. The viability of CRC cells was determined using Cell Counting Kit-8 (cat. no. C0039; Beyotime Institute of Biotechnology, Shanghai, China). Briefly, 2,000 cells/well were plated in 96-well plates. CCK-8 (10 $\mu \mathrm{l})$ was added to the culture medium. After $2 \mathrm{~h}$, the plates were assessed using a microplate reader (Biotek ELx800; BioTek Instruments, Inc., Winooski, VT, USA).

Cell migration was examined by a scratch assay. After culturing for 2 days, the cells were deprived of serum for $16 \mathrm{~h}$ and then artificially injured using a $200 \mu \mathrm{l}$ plastic pipette 
A

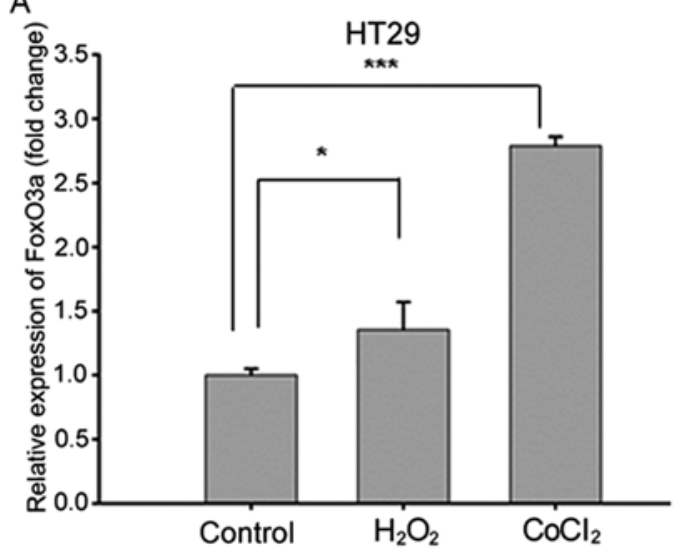

$\mathrm{C}$

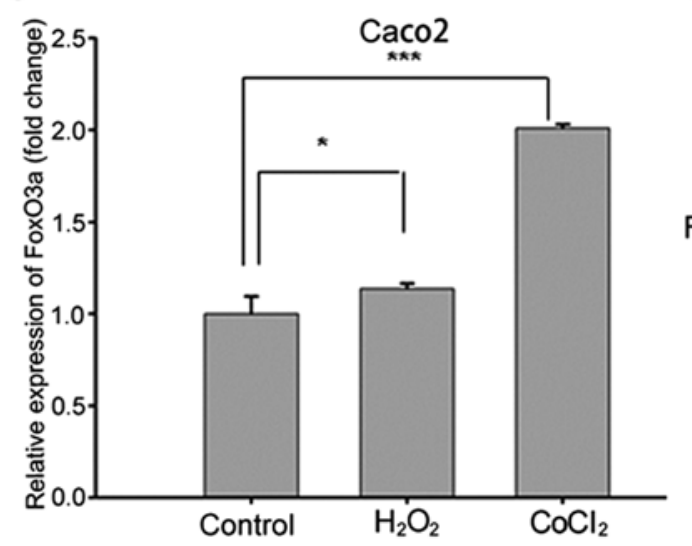

B

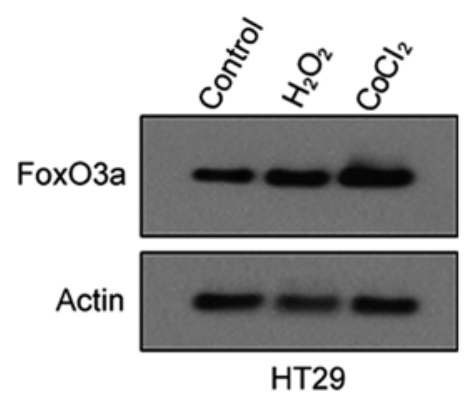

D

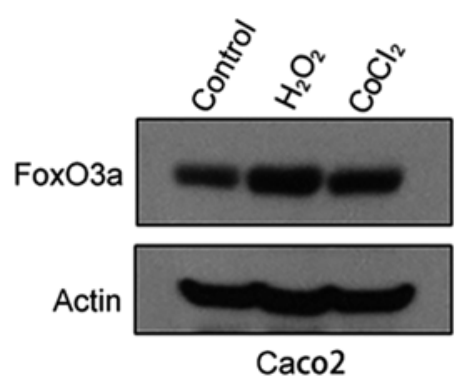

Figure 1. Stress stimulates FoxO3a transcription. (A) HT29 cells were treated with $\mathrm{CoCl}_{2}(200 \mu \mathrm{M} / 1)$ or $\mathrm{H}_{2} \mathrm{O}_{2}(200 \mu$ mol/l). FoxO3a mRNA levels were analyzed by qRT-PCR, ${ }^{*} \mathrm{P}<0.05,{ }^{* * * *} \mathrm{P}<0.001$. (B) HT29 cells were treated with $\mathrm{CoCl}_{2}(200 \mu \mathrm{M} / \mathrm{l})$ or $_{2} \mathrm{O}_{2}(200 \mu \mathrm{mol} / \mathrm{l})$. FoxO3a protein levels were analyzed by western blot analysis. (C) Caco2 cells were treated with $\mathrm{CoCl}_{2}(200 \mu \mathrm{M} / \mathrm{l})$ or $\mathrm{H}_{2} \mathrm{O}_{2}(200 \mu \mathrm{mol} / \mathrm{l})$. FoxO3a mRNA levels were analyzed by qRT-PCR, "P<0.05, ${ }_{* * * *} \mathrm{P}<0.001$. (D) Caco2 cells were treated with $\mathrm{CoCl}_{2}(200 \mu \mathrm{M} / \mathrm{l})$ or $\mathrm{H}_{2} \mathrm{O}_{2}(200 \mu \mathrm{mol} / \mathrm{l})$. FoxO3a protein levels were analyzed by western blot analysis. Data are representative of three independent experiments.

tip. Cells migrating to the front of the wound were imaged after 48 and $72 \mathrm{~h}$. The migration capacity was quantified by determining the percentage of open area using the following formula: (1-current wound size/initial wound size) x100.

The Annexin V-PE Apoptosis Detection kit (BDPharmingen; BD Biosciences, Franklin Lakes, NJ, USA) was used to analyze apoptosis according to the manufacturer's instructions. Stained cells underwent flow cytometry.

Animal studies. BALB/c nude mouse (six- to 8-week-old) weighing $\sim 18-22 \mathrm{~g}$, were obtained from SLAC Laboratory Animal Co., Ltd. (Shanghai, China). The animals were sacrificed when tumor reached $1,500 \mathrm{~mm}^{3}$ in size. HT29 cells were subcutaneously injected into the nude mice. The xenograft size was assessed twice a week and the volume was calculated using the following formula: $\mathrm{a}^{2} \mathrm{~b}^{2} / 2$ (a represents length and $\mathrm{b}$ represents width). After one month, all mice were euthanized using $\mathrm{CO}_{2}$. The tumor tissues were weighed. All animal studies were approved by the Institutional Animal Care and Use Committee of the Shanghai Institutes for Biological Sciences.

Construction of luciferase reporter vectors. The FoxO3a gene promoter-driven luciferase reporter construct was generated by inserting a 2,000 bp fragment containing the 5' flanking region of the FoxO3a gene from positions $-2,000$ to 0 into a pGL3-basic vector (Promega, Fitchburg, WI, USA). Subsequently, to identify the critical transcription activation region of the FoxO3a promoter, a series of $5^{\prime}$ deletion fragments [-1,037 bp/0, $-513 \mathrm{bp} / 0$ and $-62 \mathrm{bp} / 0]$ luciferase reporter constructs were generated by PCR using the full length FoxO3a promoter fragment as a template. All the constructs were verified by sequencing to rule out the possibility of any PCR error. The primers used to generate all constructs are listed in Table I. The putative transcription factor binding sites within FoxO3a gene promoter were screened using the TFsearch program (Kyoto University, Kyoto, Japan).

Transient transfections and luciferase assays. HT29 cells were seeded in 96-well plates and grew to a density of $80 \%$ confluency before transfection. One hundred nanograms of each luciferase reporter construct and $0.3 \mu$ l Lipofectamine 2000 (Invitrogen) reagent in Opti-MEM were transfected according to the manufacturer's instructions. In order to validate the transfection efficiency, an additional $4 \mathrm{ng}$ of pRL-TK vector which contained the Renilla luciferase gene (hRluc) under the control of the Herpes simplex virus thymidine kinase promoter was co-transfected in each well. The cells were harvested after 48-h transfection and then lysed with $20 \mu \mathrm{l}$ passive lysis buffer for each well (Promega). Luciferase activity was determined using Dual-Luciferase Reporter Assay system (Promega) on 
A

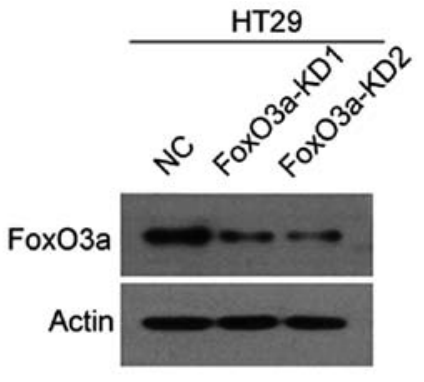

C NC

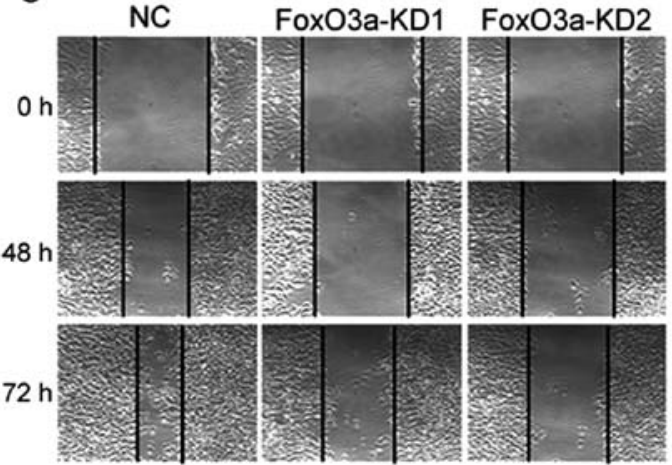

E

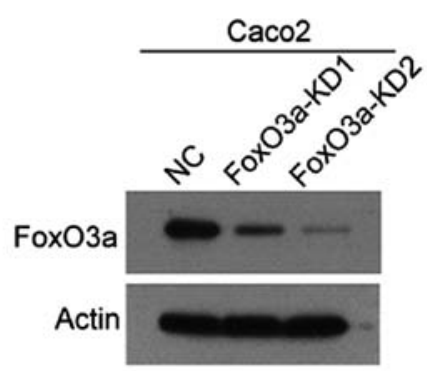

B $2.5 \rightarrow$ HT29 GFP.NC

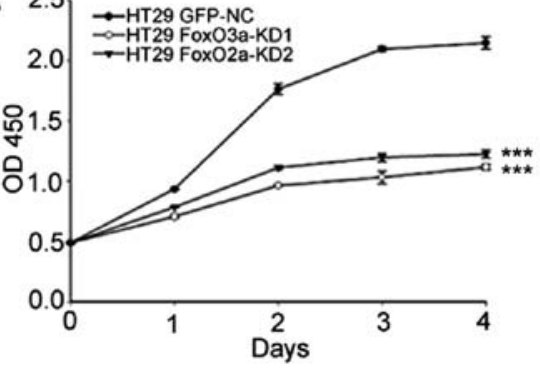

D

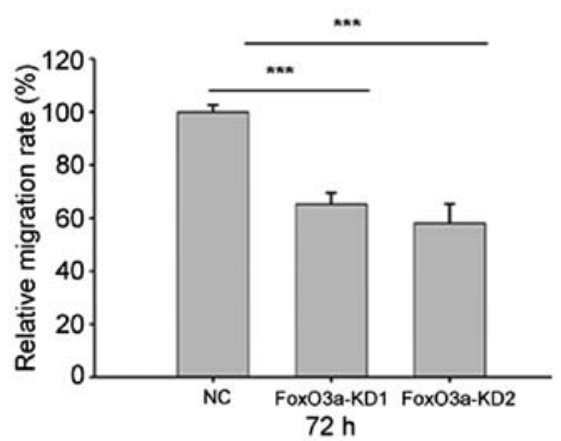

$\mathrm{F}$

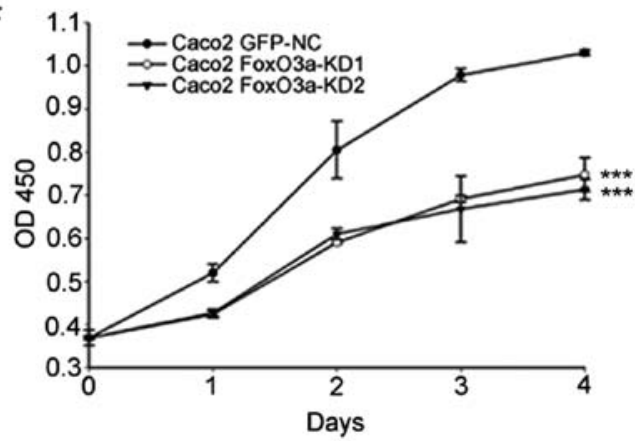

Figure 2. Knockdown of FoxO3a reduces CRC cell proliferation and migration ability. (A) The expression of FoxO3a in HT29 cells with FoxO3a knockdown or NC was detected by western blotting. (B) CCK-8 analysis of HT29 cells with FoxO3a knockdown or NC, ${ }^{* * *} \mathrm{P}<0.001$. (C and D) Scratch analysis of cell migration ability in HT29 cells with or without FoxO3a knockdown, ${ }^{* * *} \mathrm{P}<0.001$. (E) The expression of FoxO3a in Caco2 cells with FoxO3a knockdown or NC was detected by western blotting. (F) CCK-8 analysis of Caco2 cells with FoxO3a knockdown or NC, ${ }^{* * * *} \mathrm{P}<0.001$.

a luminescence reader (AccuFLEX Lumi 400; Aloka, Tokyo, Japan). Each expression vector was mixed with the luciferase vectors at 1:2 concentration ratio. All the experiments were repeated at least three times and the results were expressed as the mean \pm standard deviation (SD).

Statistical analysis. In the present study, data are presented as the mean \pm SD of three independent experiments. Two-tailed Student's t-test was used to analyze the differences between groups using GraphPad Prism version 5.0 (GraphPad Software Inc., La Jolla, CA, USA). $\mathrm{P}<0.05$ was considered to indicate a statistically significant difference.

\section{Results}

Upregulated FoxO3a expression in CRC cell lines after stress stimuli. In order to elucidate the function of FoxO3a in CRC under stress stimuli, we investigated the expression levels of FoxO3a in HT29 and Caco2 cell lines treated with $\mathrm{H}_{2} \mathrm{O}_{2}$ or $\mathrm{CoCl}_{2}$ using qRT-PCR and western blot analysis. Both the qRT-PCR and western blotting results revealed that the expression of FoxO3a increased after oxidative stress stimuli treatment (Fig. 1), indicating that FoxO3a transcription was upregulated in response to hypoxia and oxidative stress.

Knockdown of FoxO3a reduces $\mathrm{CRC}$ cell proliferation and migration ability. We stably downregulated FoxO3a using lentivirus-mediated shRNA. The effect of FoxO3a-knockdown in HT29 cells was verified by western blot analysis (Fig. 2A). CCK-8 analysis revealed that knockdown of FoxO3a in HT29 cells reduced cell proliferation (Fig. 2B). Scratch analysis indicated that inhibition of FoxO3a also suppressed cell migration in HT29 cells (Fig. 2C and D). Subsequently we knocked down FoxO3a in Caco2 cells (Fig. 2E). Consistently, CCK-8 analysis revealed that FoxO3a-knockdown suppressed cell proliferation in Caco2 cells (Fig. 2F). These results indicated that FoxO3a was important for CRC cell proliferation and migration.

Knockdown of FoxO3a sensitizes CRC cells to cetuximab and combination treatment. In order to elucidate the func- 
A

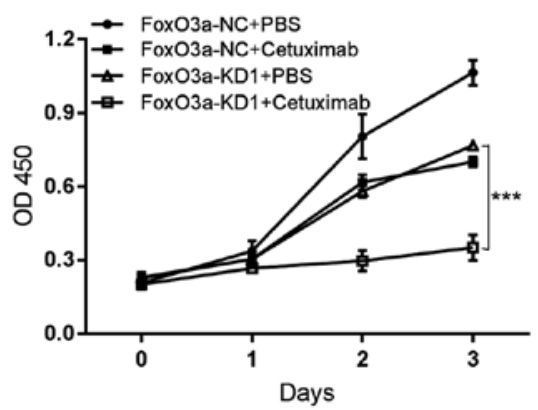

C

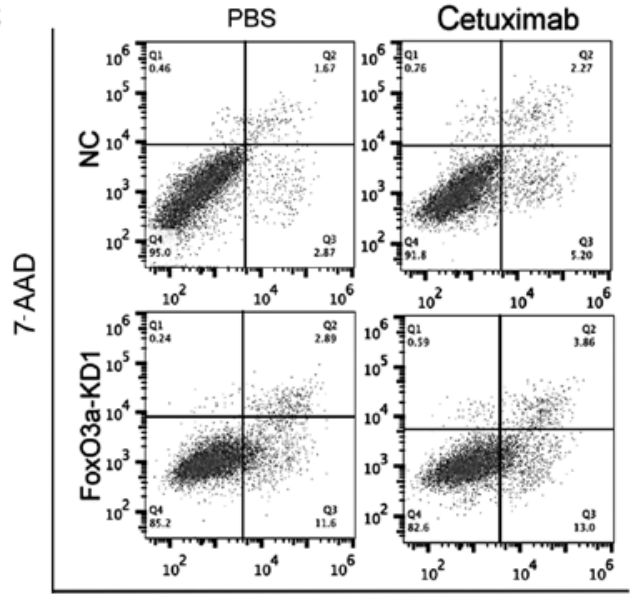

PE-Annexin V

E

HT29 (cetuximab+irinotecan)

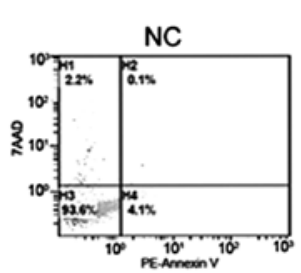

B

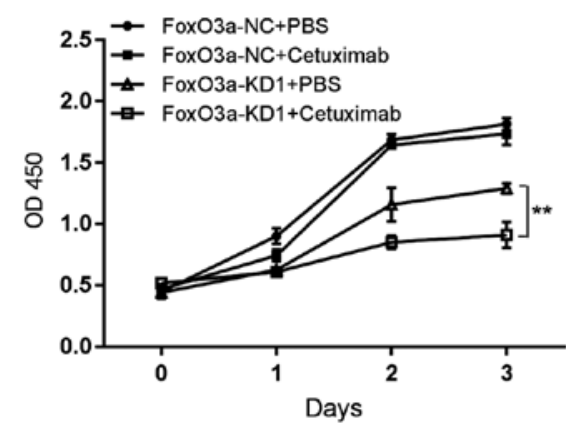

D

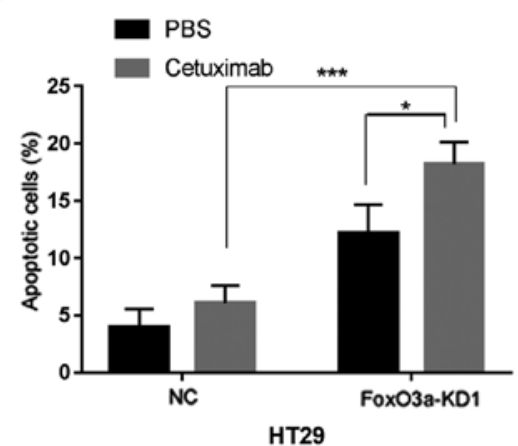

Figure 3. Knockdown of FoxO3a sensitizes CRC cells to cetuximab and combination treatment. (A) CCK-8 analysis of the Caco2 cells after cetuximab treatment in FoxO3a-knockdown or NC group, ${ }^{* * *} \mathrm{P}<0.001$. (B) CCK-8 analysis of the HT29 cells after cetuximab treatment in FoxO3a-knockdown or NC group, ${ }^{* *} \mathrm{P}<0.01$. (C and D) FACS analysis of cell apoptosis in HT29 cells with or without FoxO3a-knockdown treated with cetuximab. ${ }^{*} \mathrm{P}<0.05$, ${ }^{* * * *} \mathrm{P}<0.001$. (E and F) FACS analysis of cell apoptosis in HT29 cells with or without FoxO3a-knockdown treated with cetuximab combined with irinotecan. ${ }^{* * *} \mathrm{P}<0.001$. $\mathrm{CRC}$, colorectal cancer.

tion of FoxO3a in CRC therapy, we treated these cells with cetuximab alone or combined with irinotecan (Fig. 3). CCK-8 analysis indicated that knockdown of FoxO3a in $\mathrm{Caco} 2$ and HT29 cells reduced cell proliferation after treatment with cetuximab (Fig. 3A and B). Apoptotic analysis using Annexin V/7-AAD revealed that knockdown of FoxO3a promoted the apoptosis of HT29 cell lines treated with cetuximab (Fig. 3C and D). Knockdown of FoxO3a also increased cell apoptosis after treatment of cetuximab combined with irinotecan (Fig. 3E and F). In conclusion, our data indicated that FoxO3a may participate in CRC response to cetuximab treatment.

Inhibition of FoxO3a reduces CRC progression in vivo. After subcutaneously inoculating HT29 cells with or without knockdown of FoxO3a in a xenograft animal model, we observed that the tumorigenesis ability of the FoxO3a-knockdown group was significantly reduced compared with the NC group. Both tumor volume and weight were significantly decreased in FoxO3a-knockdown group compared with NC group (Fig. 4). In conclusion, these findings indicated an important role of FoxO3a in CRC progression.

Analysis of putative transcription factor binding sites in FoxO3a gene promoter. The transcription factor binding sites of the FoxO3a gene promoter was analyzed using TFsearch program. The results revealed that many potential SP1 and AP2 transcription factor binding sites were found within the FoxO3a promoter (Fig. 5A). Furthermore, most of them were centered on regions $-2,000 /-1,032$ bp and $-513 /-62$ bp (Fig. 5A). In addition, some other transcription factor binding sites were also identified, such as C/EBP $\beta$, NF-1, AP-1, SRF and NRF-1. 


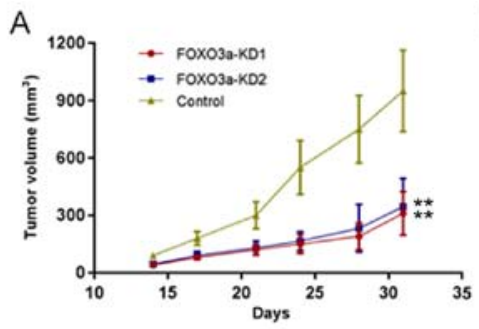

C

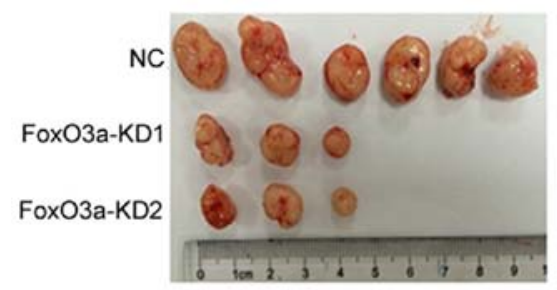

B

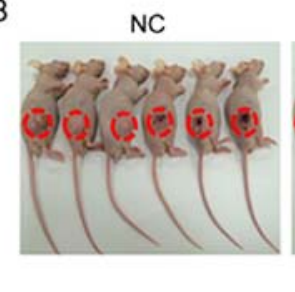

FoxO3a-KD1

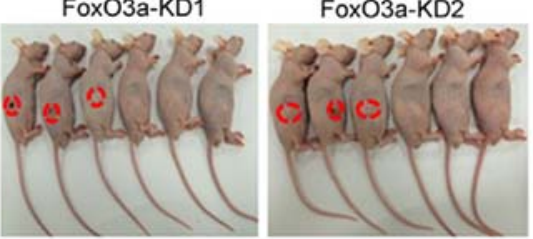

D

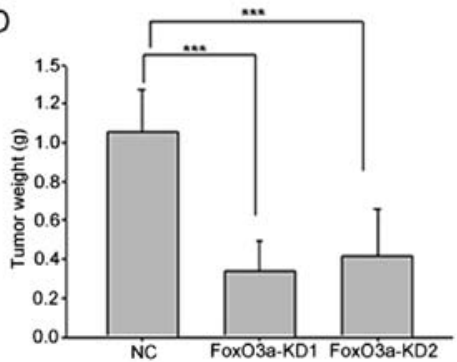

Figure 4. Inhibition of FoxO3a reduces tumorigenesis of colorectal cells in vivo. (A) The volume of xenografts derived from subcutaneously inoculated HT29 cells with or without FoxO3a-knockdown, ${ }^{* *} \mathrm{P}<0.01$. (B) Image of the animal models. (C) Image of the xenografts. (D) The weight of xenografts derived from subcutaneously inoculated FoxO3a-knockdown or NC HT29 cells. ${ }^{* * *} \mathrm{P}<0.001$.

To ascertain the regulation of the FoxO3a gene transcription, full length of FoxO3a gene promoter $(-2,000 \mathrm{bp} / 0)$ and a series of 5'deletion fragment [-1,032 bp/0,-513 bp/0,-62 bp/0] luciferase reporter plasmids were constructed.

Activity analysis of FoxO3a promoter luciferase reporter constructs. The activity of different lengths of FoxO3a promoter luciferase reporter constructs was analyzed in HT29 cell lines. As displayed in Fig. 5B, the percentage of activity reduction was $>80 \%$ in deletion construct $-1,032 \mathrm{bp} / 0$ compared to wild-type (WT) $(-2,000 \mathrm{bp} / 0)$. A similar phenomenon was observed between deletion constructs $-513 \mathrm{bp} / 0$ and $-62 \mathrm{bp} / 0$, accounting for the remaining $20 \%$ transcriptional activity. Thus, these results indicated that the regions between $-2,000 /-1,032$ bp and -513/-62 bp of FoxO3a promoter play an important role in regulating the transcription of $\mathrm{FoxO} 3 a$ gene.

According to Fig. 5A, there were plenty of SP1 and AP2 transcription factor binding sites between regions $-2,000 /-1,032 \mathrm{bp}$ and $-513 /-62 \mathrm{bp}$. These results indicated that SP1 and AP2 may play important roles in FoxO3a gene transcription. In order to further identify the major transcriptional factor, we transiently co-transfected the FoxO3a promoter luciferase reporter constructs individually combined with SP1 or AP2 expression plasmids. The expression of SP1 was examined by western blot analysis (Fig. 5C). It was revealed that the activity of FoxO3a promoter luciferase constructs co-transfected with SP1 expression plasmid was obviously increased, except $-62 \mathrm{bp} / 0$ construct. Furthermore, luciferase activity of constructs containing $-513 \mathrm{bp} / 0$ and $-1032 \mathrm{bp} / 0$ region of FoxO3a promoter increased almost 1.5 times when co-transfected with SP1 expression plasmid, compared with 2.5 times of increase in WT (Fig. 5D and E). However, co-transfection with AP2 did not promote FoxO3a promoter luciferase activity (Fig. 6). These results confirmed that $-2,000 /-1,032$ bp and -513/-62 bp regions of FoxO3a promoter were the critical regions for FoxO3a gene transcription and that the transcription factor SP1 was an important regulator for FoxO3a gene transcription.

\section{Discussion}

In the present study, we characterized the transcriptional activity and regulation of the 5 'flank of human FoxO3a gene located in the -2,000/-1,032 bp and -513/-62 bp regions. This study demonstrated that the SP1 site plays an important role in regulating the transcription of the FoxO3a gene in CRC cells. Furthermore, we found that the FoxO3a gene was upregulated in response to stress conditions, hypoxia and oxidative stress and that the upregulated FoxO3a gene was important for CRC progression in vivo and in vitro. These findings may help to understand the complicated pathological function of FoxO3a gene in tumor biology.

FoxO3a gene has an ambiguous function in CRC. A study revealed that FoxO3a inactivation promoted tumor progression (19). However, there are also studies indicating that FoxO3a protected cells under stress conditions, including oxidative stress, serum deprivation and hypoxia (20). Consistent with these studies, we found that the expression of FoxO3a in CRC cells was increased significantly in response to oxidative stress and hypoxia. However, the precise function of $\mathrm{FoxO} 3 \mathrm{a}$ in tumor progression and tumor microenvironment remains elusive. FoxO3a was found to promote invasion of cancer cells (12). Therefore, caution should be taken when FoxO3a is employed as a potential target for cancer therapy.

The ubiquitous transcription factor SP1 is involved in the regulation of many genes (21-23). Although the expression of SP1 can be detected in most cells, its expression varied during development $(24,25)$. SP1 is the founding member of SP/XKLF proteins, which contain three highly homologous C-terminal zinc-finger motifs and are capable of binding similar DNA sequences. The combination of SP/XKLF proteins can either compete or interact with a given promoter element to activate or suppress the transcription (21). Therefore, it is not easy to confirm the function of SP1, due toå the potential functions of its related family members. However, SP1 is essential for embryogenesis, because SP1-/- mouse embryos display growth 
A

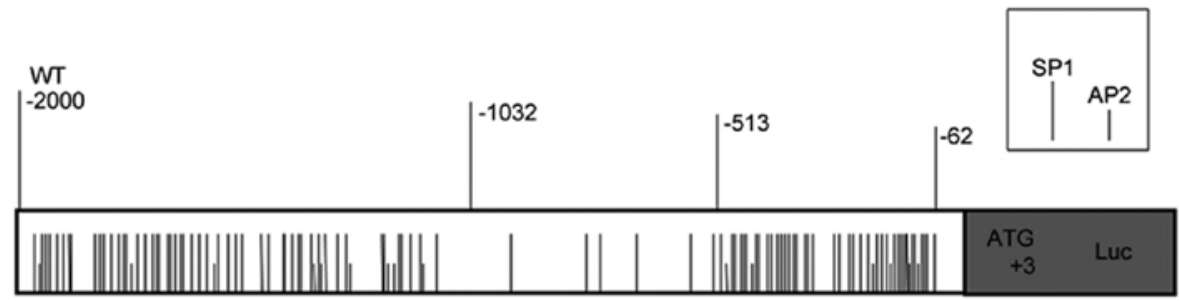

B

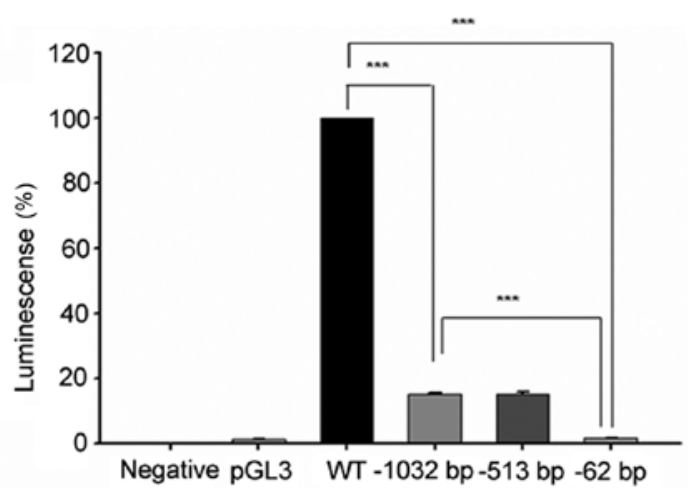

D

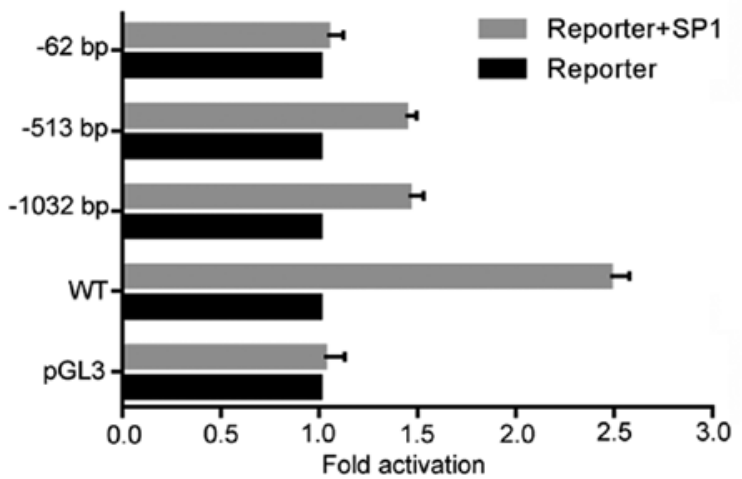

C

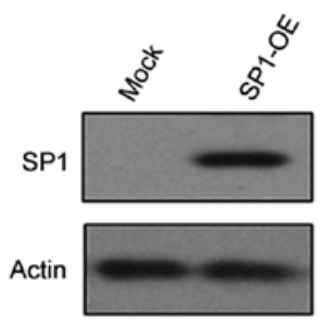

E

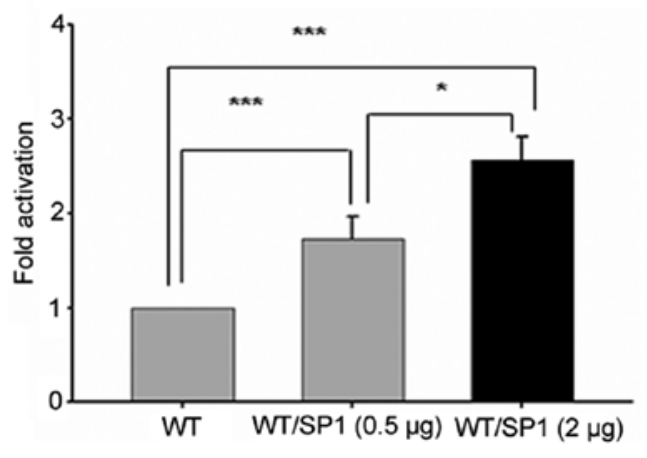

Figure 5. Analysis of putative transcription factor binding sites in FoxO3a gene promoter. (A) Sequences approximately 2000 bp upstream the translation start codon of the FoxO3a gene. TFsearch program was used to identify putative SP1 and AP2 transcription factor binding sites. Longer line indicates SP1, shorter line indicates AP2. WT, wild-type. (B) -2,000 bp-luc, $-1,032$ bp-luc, -513 bp-luc and - 62 bp-luc fragments were inserted into the pGL3-basic vectors. HT29 cells were transfected with the constructs. The results are presented as the relative luciferase activity compared with wild-type (WT). ${ }^{* * *} \mathrm{P}<0.001$. (C) Overexpression (OE) of SP1 was examined by western blot analysis. (D) The pGL3-basic vector constructs containing fragments of various lengths were transfected or co-transfected with the SP1 expression vectors into HT29 cells. For the co-transfection experiments, the luciferase reporter constructs and SP1 expression vector were used at 1:2 concentration ratio. The relative luciferase activity is presented in each group. (E) The pGL3-basic vector constructs containing $-2,000$ bp were transfected or co-transfected with the increasing SP1 expression vectors into HT29 cells. The relative luciferase activity is presented. ${ }^{*} \mathrm{P}<0.05$, ${ }^{* * * *} \mathrm{P}<0.001$.
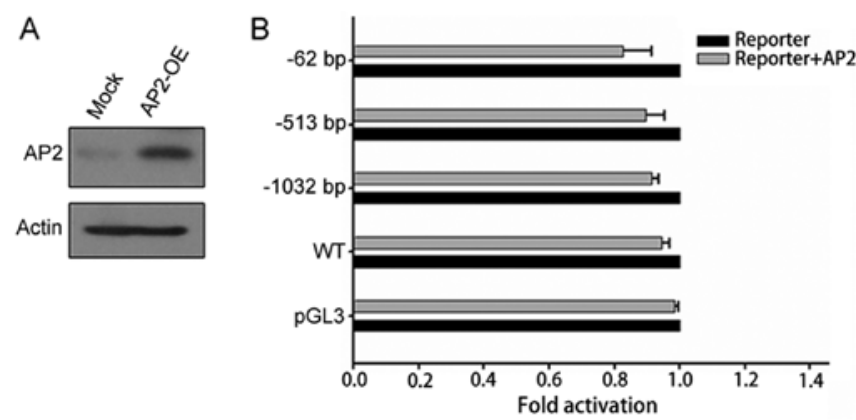

Figure 6. Promoter activity of the deletion regions with the AP2 overexpression. (A) The expression of AP2 was examined by western blot analysis. (B) The pGL3-basic vector constructs containing fragments of various lengths were transfected or co-transfected with the AP2 expression vectors into HT29 cells. For the co-transfection experiments, the luciferase reporter constructs and factor expression vector were used at 1:2 concentration ratio. The relative luciferase activity is presented.

retardation and die during gestation (26). In the present study, we found that SP1 was the crucial regulator of FoxO3a tran- scription in CRCs. More transcriptional factors need to be identified in this complex regulation network. 
In conclusion, our findings determined that the crucial regions corresponding to the SP1 binding sites located between $-2,000$ and $-1,037$ bp were essential for FoxO3a transcriptional activity. Furthermore, FoxO3a transcription was upregulated in response to hypoxic and oxidative stress in colorectal tumor cells, indicating that the interaction between SP1 and FoxO3a may have important implications in CRC progression.

\section{Acknowledgements}

Not applicable.

\section{Funding}

The present study was funded by the National Natural Science Foundation of China (no. 81602038), the Shanghai Science and Technology Commission (no. 14ZR1406500) and the National Natural Science Foundation of China (no. 81502003).

\section{Availability of data and materials}

The datasets used during the present study are available from the corresponding author upon reasonable request.

\section{Authors' contributions}

YY and TL conceived and designed the experiments; YY, HL, SY and LL performed the experiments; YY, KP and HL analyzed the data; RZ, YW and WL contributed to reagents/materials/analysis tools; YY, KP wrote the paper. All authors read and approved the manuscript and agree to be accountable for all aspects of the research in ensuring that the accuracy or integrity of any part of the work are appropriately investigated and resolved.

\section{Ethics approval and consent to participate}

All animal studies were approved by the Institutional Animal Care and Use Committee of the Shanghai Institutes for Biological Sciences.

\section{Consent for publication}

Not applicable.

\section{Competing interests}

The authors declare that they have no competing interests.

\section{References}

1. Torre LA, Bray F, Siegel RL, Ferlay J, Lortet-Tieulent J and Jemal A: Global cancer statistics, 2012. CA Cancer J Clin 65 87-108, 2015.

2. Gatenby RA and Gillies RJ: A microenvironmental model of carcinogenesis. Nat Rev Cancer 8: 56-61, 2008.

3. Albini A and Sporn MB: The tumour microenvironment as a target for chemoprevention. Nat Rev Cancer 7: 139-147, 2007.

4. RosichL, Saborit-Villarroya I,López-Guerra M,Xargay-Torrent S, Montraveta A, Aymerich M, Villamor N, Campo E, Pérez-Galán P, Roué G, et al: The phosphatidylinositol-3-kinase inhibitor NVP-BKM120 overcomes resistance signals derived from microenvironment by regulating the $\mathrm{Akt} / \mathrm{FoxO} 3 \mathrm{a} / \mathrm{Bim}$ axis in chronic lymphocytic leukemia cells. Haematologica 98: 1739-1747, 2013.
5. Oellerich MF and Potente M: FOXOs and sirtuins in vascular growth, maintenance, and aging. Circ Res 110: 1238-1251, 2012.

6. Becker T, Loch G, Beyer M, Zinke I, Aschenbrenner AC, Carrera P, Inhester T, Schultze JL and Hoch M: FOXO-dependent regulation of innate immune homeostasis. Nature 463: 369-373, 2010.

7. Monsalve $\mathrm{M}$ and Olmos Y: The complex biology of FOXO. Curr Drug Targets 12: 1322-1350, 2011.

8. Hedrick SM, Hess Michelini R, Doedens AL, Goldrath AW and Stone EL: FOXO transcription factors throughout T cell biology. Nat Rev Immunol 12: 649-661, 2012.

9. Seiler F, Hellberg J, Lepper PM, Kamyschnikow A, Herr C, Bischoff M, Langer F, Schäfers HJ,Lammert F, Menger MD, et al: FOXO transcription factors regulate innate immune mechanisms in respiratory epithelial cells. J Immunol 190: 1603-1613, 2013.

10. He L, Yang X, Cao X, Liu F, Quan M and Cao J: Casticin induces growth suppression and cell cycle arrest through activation of FOXO3a in hepatocellular carcinoma. Oncol Rep 29: 103-108, 2013.

11. Yung MM, Chan DW, Liu VW, Yao KM and Ngan HY: Activation of AMPK inhibits cervical cancer cell growth through AKT/FOXO3a/FOXM1 signaling cascade. BMC Cancer 13: 327, 2013.

12. Bullock MD, Bruce A, Sreekumar R, Curtis N, Cheung T, Reading I, Primrose JN, Ottensmeier C, Packham GK, Thomas G and Mirnezami AH: FOXO3 expression during colorectal cancer progression: Biomarker potential reflects a tumour suppressor role. Br J Cancer 109: 387-394, 2013.

13. Tenbaum SP, Ordóñez-Morán P, Puig I, Chicote I, Arqués O, Landolfi S, Fernández Y, Herance JR, Gispert JD, Mendizabal L, et al: $\beta$-catenin confers resistance to $\mathrm{PI} 3 \mathrm{~K}$ and $\mathrm{AKT}$ inhibitors and subverts FOXO3a to promote metastasis in colon cancer. Nat Med 18: 892-901, 2012.

14. Zhao Y, Zhang W, Guo Z, Ma F, Wu Y, Bai Y, Gong W, Chen Y, Cheng T, Zhi F, et al: Inhibition of the transcription factor Sp1 suppresses colon cancer stem cell growth and induces apoptosis in vitro and in nude mouse xenografts. Oncol Rep 30: 1782-1792, 2013.

15. Kim DW, Ko SM, Jeon YJ, Noh YW, Choi NJ, Cho SD, Moon HS, Cho YS, Shin JC, Park SM, et al: Anti-proliferative effect of honokiol in oral squamous cancer through the regulation of specificity protein 1. Int J Oncol 43: 1103-1110, 2013.

16. Jeon YJ, Ko SM, Cho JH, Chae JI and Shim JH: The HDAC inhibitor, panobinostat, induces apoptosis by suppressing the expresssion of specificity protein 1 in oral squamous cell carcinoma. Int J Mol Med 32: 860-866, 2013.

17. Karin M, Liu Z and Zandi E: AP-1 function and regulation. Curr Opin Cell Biol 9: 240-246, 1997.

18. Livak KJ and Schmittgen TD: Analysis of relative gene expression data using real-time quantitative PCR and the $2^{-\Delta \Delta C_{\mathrm{T}}}$ method. Methods 25: 402-408, 2001.

19. Greer EL and Brunet A: FOXO transcription factors at the interface between longevity and tumor suppression. Oncogene 24: 7410-7425, 2005.

20. Li Z, Zhang H, Chen Y, Fan L and Fang J: Forkhead transcription factor FOXO3a protein activates nuclear factor $\kappa \mathrm{B}$ through B-cell lymphoma/leukemia 10 (BCL10) protein and promotes tumor cell survival in serum deprivation. J Biol Chem 287: 17737-17745, 2012.

21. Philipsen S and Suske G: A tale of three fingers: The family of mammalian $\mathrm{Sp} / \mathrm{XKLF}$ transcription factors. Nucleic Acids Res 27: 2991-3000, 1999.

22. Li L, He S, Sun JM and Davie JR: Gene regulation by Sp1 and Sp3. Biochem Cell Biol 82: 460-471, 2004.

23. Schreiber M, Baumann B, Cotton M, Angel P and Wagner EF: Fos is an essential component of the mammalian UV response. EMBO J 14: 5338-5349, 1995.

24. Lania L, Majello B and De Luca P: Transcriptional regulation by the Sp family proteins. Int J Biochem Cell Biol 29: 1313-1323, 1997.

25. Suske G: The Sp-family of transcription factors. Gene 238: 291-300, 1999.

26. Marin M, Karis A, Visser P, Grosveld F and Philipsen S: Transcription factor Sp1 is essential for early embryonic development but dispensable for cell growth and differentiation. Cell 89: 619-628, 1997. 